\title{
Upsurge in BCG Vaccine Induced lymphadenitis: Case Series
}

\author{
Manju V' ${ }^{1}$ Amrita Parida², Asha Hegde' ${ }^{1}$ Phalguna Kousikakatakam ${ }^{3}$ \\ 'Department of Paediatrics, Melaka Manipal Medical College, Manipal Academy of Higher Education, Manipal, Karnataka, INDIA \\ 2Department of Pharmacology, Kasturba Medical College, Manipal Academy of Higher Education, Mangalore, Karnataka, INDIA. \\ ${ }^{3}$ Department of Paediatrics, Kasturba Medical College, Manipal Academy of Higher Education, Manipal, Karnataka, INDIA.
}

\begin{abstract}
Introduction: Tuberculosis is one of the serious healthcare concerns of the world today. The only vaccine available for tuberculosis till date is the BCG vaccine. It is one of the safest vaccine and is less commonly associated with adverse effects. However, recently we have seen an upsurge in number of BCG vaccine associated lymphadenitis in our hospital. Objective: To report the increase in number of BCG associated lymphadenitis cases in our hospital. Materials and methods: A total of ten cases of BCG associated lymphadenitis were seen in our hospital in the past one year. (May 2016 to April 2017) Data related to vaccination and the course of lymphadenitis was collected from the parents. Observations/Results: Out of the ten cases, 3 were vaccinated outside while the remaining infants were vaccinated in our hospital. Three out of the ten infants required intervention and remaining 7 were reassured and followed up, the swelling subsided in most of them. The most probable cause of the increased incidence in our hospital could be because of a particular batch
\end{abstract}

of vaccine containing an altered strain/ change in the number of viable organism. Conclusion: We are reporting the cases as it might lead to an awareness among other practitioners and will also provide a scope for further studies. Increased surveillance and appropriate treatment guidelines for BCG vaccine associated lymphadenitis is the need of the hour.

Key words: Tuberculosis, BCG vaccine, Iymphadenitis

Correspondence:

Dr. Amrita Parida, Assistant Professor, Department of Pharmacology, Kasturba Medical College, Manipal Academy of Higher Education, Light House hill road, Mangalore, Karnataka 575001, INDIA.

Phone: 08242422271

Email: amrita_parida@yahoo.com

DOI: 10.5530/jyp.2018.10.27

\section{INTRODUCTION}

Tuberculosis (TB) is one of the major health problems in the current era. As per the WHO data, 10.4 million people were affected with TB and 1.8 million deaths due to TB in the year 2015. One million children (aged 0-14 years) fell ill with TB and 17,000 deaths were reported. ${ }^{1}$ The emerging multidrug resistant strains of $\mathrm{TB}$ bacilli is the prime concern related to treatment of TB. As a preventive measure, the only vaccine currently available is the BCG vaccine. There are few other vaccines which are currently under development. BCG vaccine prevents the disseminated forms of tuberculosis in children, including TB meningitis. It is one of the most widely used vaccine, reaching about more than $80 \%$ of neonates in countries where it is a part of the national immunization programme. In India, under the Expanded Programme of Immunization (EPI), BCG vaccine is given to all newborns at birth. There is no provision for booster doses under the programme. The various complications of BCG vaccine include regional suppurative/non suppurative lymphadenitis, eczema vaccinatum, lupoid reactions osteomyelitis/ostitis and disseminated TB. Regional lymphadenitis is one of the common complications of BCG vaccine. However, in the past one year, we have noticed an increase in the number of BCG induced lymphadenitis in our hospital. Hence, we thought of reporting these 10 patients who presented to our outpatient department to create an increased awareness among the health care providers.

\section{MATERIALS AND METHODS}

We collected the data of 10 infants with signs of lymphadenopathy due to BCG vaccine who came to the paediatric outpatient department of Dr. TMA Pai Rotary Hospital, Karkala, Karnataka, India. Since data was collected retrospectively, it was not possible to take informed consent.
Ethics committee clearance: Since it a case series and article does not reveal any personal information/identity of patients, ethics committee approval was not sought. Permission to publish the data has been taken from the concerned higher authorities.

\section{RESULTS}

We are reporting 10 cases of BCG vaccine associated lymphadenitis seen in our hospital in the past one year. Out of the ten cases, 3 were vaccinated outside whereas the remaining infants were vaccinated in our hospital. Three out of the ten patients required intervention as mentioned in table no. 1. Remaining 7 patients were reassured and followed up, the swelling subsided in most of them.

\section{DISCUSSION}

BCG vaccine associated lymphadenitis is defined as the development of ipsilateral regional lymoh node enlargement after BCG vaccination and absence of local/ systemic signs of inflammation. Lymphadenitis can be of two types: 1) simple/ non suppurative- needs no intervention and patient's parents have to be assured and it usually resolves on its own or sometimes may turn into suppurative form. 2) suppurative - this is a serious form. It can lead to rupture, sinus and later scar formation. To prevent these complications, sometimes surgical excision might be required.

In our hospital, BCG vaccine is administered intradermally. We have reported these cases because we found an increase in the number of cases of BCG associated lymphadenopathy in the past one year. There were 10 cases from May 2016 to April 2017. We had not seen any case of BCG induced lymphadenitis in the past 5 years in our hospital prior to these 


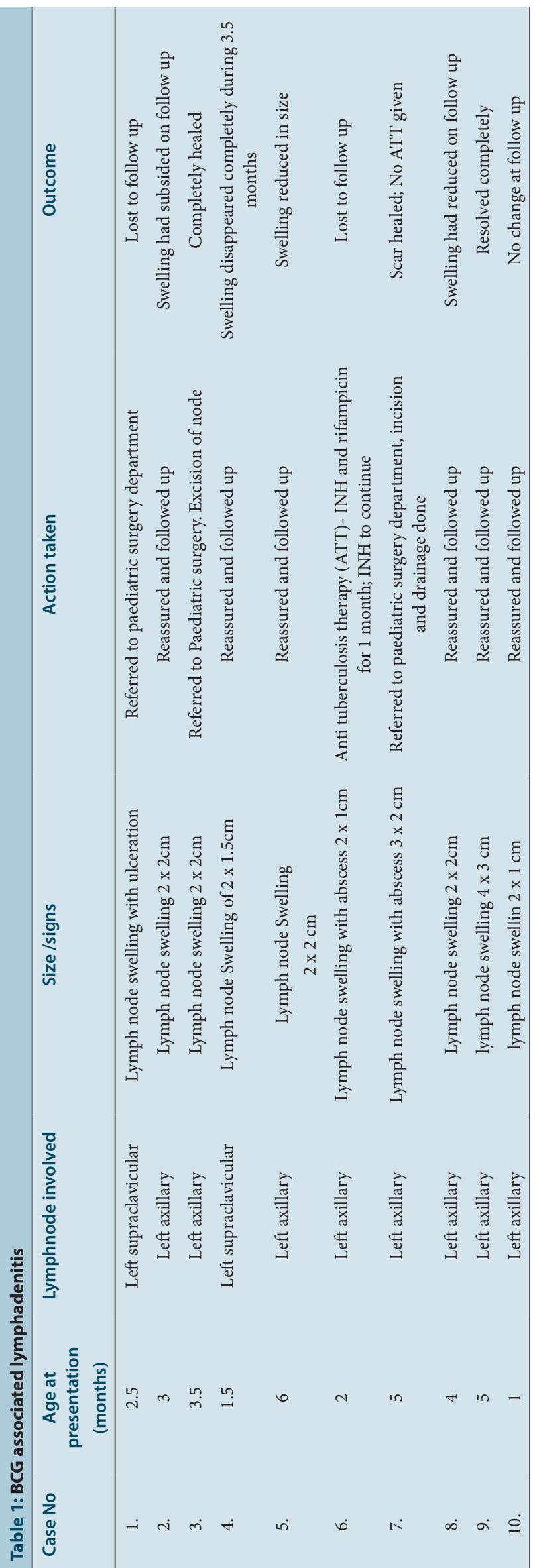

cases. Information prior to that is not available. The various risk factors associated with BCG related lymphadenitis includes (i) Vaccine related factors: BCG strain used in the vaccine, dose, viability of organisms in the vaccine, skill of the personnel administering the vaccine. (ii) Host factors: age at vaccination, immunological response to vaccine, characteristics of recipient population. ${ }^{2}$

Of the factors mentioned above the probable cause of the increased incidence in our hospital could be the vaccine related (a particular batch of vaccine containing an altered strain/ number of viable organism). The vaccine used in our hospital is provided by the government of India under the national immunization programme. There was an incident of BCG induce lymphadenitis outbreak in Malaysia because of change in the strain of bacilli used in vaccine preparation. ${ }^{3}$ In the study they have mentioned that the increase in the incidence was due to the change in the vaccine strain from Tokyo-172 to Pasteur-1173. The authors also found that the Pasteur strain BCG vaccine had altered count of viable organisms. The incidence of lymphadenitis declined after the Tokyo strain was reintroduced. Glaxo 1077 and Tokyo 172 are "weak" strains, whereas Pasteur 1173 and Danish1331 are "strong" strains. The incidence of lymphadenitis is higher with strong strains. We have not checked on the viable count of the BCG vaccine used to immunize infants in our hospital, hence cannot comment on this aspect. The other factors such as immunization technique, dose and the skill of the vaccine provider is probably not a reason because vaccine in our hospital is provided by trained nurses since the past two decades.

The concern with BCG lymphadenitis is that there is no clarity regarding the treatment of BCG induced lymphadenitis. Some physicians use antibiotics like erythromycin and antitubercular drugs and certain studies have shown them to be effective. ${ }^{4,5,6}$ However controlled studies have suggested that use of antibiotics neither reduces the risk of suppuration nor does it speed up healing. ${ }^{7,89,10}$ Since non suppurative lymphadenitis usually heals on its own, it is better not to start antibiotics as they have their own side effects. ${ }^{11}$ For suppurative lymphadenitis, it is advocated that needle aspiration causes rapid healing and prevents complications such as sinus and scar formation. Surgical excision is sometimes advised when needle aspiration fails as in case of mature and multiloculated lymphnodes or when there is already a sinus formation. These treatments are the suggestions of various authors based on their observations and studies, however there is lack of treatment guidelines for BCG induced lymphadenitis.

The other problem includes lack of clear case definition of BCG associated lymphadenitis. An unambiguous case definition would help the health care providers to identify the cases correctly. There is also a need for increase in the surveillance for detection of cases because most of these cases might go undetected as they usually subside without any medical intervention. The vaccine purity check should also be made more stringent emphasizing on the control of viability of organisms.

\section{CONCLUSION}

A recent increase in the number of cases of BCG vaccine induced lymphadenitis in our hospital was seen over past few months, the reason for which is still unknown. We would encourage other health care professionals to report similar cases. Further detailed studies needs to be conducted to find out exact cause of increased incidence in cases and the most appropriate way to deal with it. BCG vaccine is administered to more than $80 \%$ of infants in our country hence increase in the incidence of adverse effects is a matter of grave concern as this might have detrimental effects on attitude of parents towards vaccination.

\section{CONFLICTS OF INTEREST}

The authors declare no conflict of interest. 


\section{Funding source- None}

Contribution of authors: All the four authors were actively involved in data collection, analysis, framing and final editing of the manuscript.

\section{REFERENCES}

1. World Health Organization. Tuberculosis fact sheet no.104. [Internet] 2017 [cited 2017 May 09]. Available from http://www.who.int/mediacentre/factsheets/ fs104/en/index.htm

2. Goraya JS, Virdi VS. Bacille Calmette-Guérin lymphadenitis. Postgraduate Medical Journal. 2002;78:327-9

3. Hooi LN, Athiyah SO. An outbreak of BCG related lymphadenitis in Malaysian infants. Med J Malaysia. 1994;49(4):327-35.

4. Souza MGR, Sant ACC, Silva LJR, et al. Intradermal BCG vaccination complications-analysis of 51 cases. Tubercle. 1983;64:23-7

5. Power JT, Stewart IC, Ross JD. Erythromycin in the management of trouble- some BCG lesions. Br J Dis Chest. 1984;78:192-4.

6. Rezai MS, Khotaei G, Mamishi S, Kheirkhah M, Parvaneh N. Disseminated Bacillus Calmette-Guerin infection after BCG vaccination. J Trop Pediatr. 2008:54(6):413-6

7. Caglayan S, Yegin O, Kayran K, et al. Is medical therapy effective for regional lymphadenitis following BCG vaccination?. Am J Dis Child. 1987;141:1213-4.

8. Kuyucu N, Kuyucu S, ícal B, et al. Comparison of oral erythromycin, local administration of streptomycin and placebo therapy for non-suppurative Bacillus Calmette-Guérin lymphadenitis. Pediatr Infect Dis J. 1998;17:524-5.

9. Noah PK, Pande D, Johnson B, et al. Evaluation of oral erythromycin and local isoniazid instillation therapy in infants with Bacillus Calmette-Guerin lymphadenitis and abscesses. Pediatr Infect Dis J1993;12:136-9.

10. Close GC, Nasiiro R. Management of BCG adenitis in infancy. J Trop Pediatr 1985:31:286.

11. G KK, C FY. Brief Communication BCG Adenitis - Need for Increased Awareness. $2011 ; 18(2): 66-9$

Article History: Submission Date : 01-09-2017 ; Revised Date : 02-10-2017; Acceptance Date : 12-11-2017.

Cite this article: Manju V, Parida A, Hegde A, Kousikakatakam P. Upsurge in BCG vaccine induced lymphadenitis: Case series. JYoung Pharm. 2018;10(1):123-5. 